\title{
A new laboratory-based surveillance system (Respiratory DataMart System) for influenza and other respiratory viruses in England: results and experience from 2009 to 2012
}

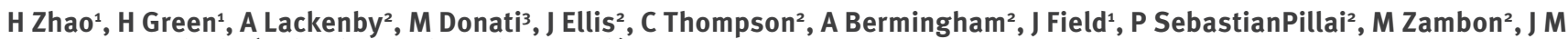
Watson ${ }^{1}$, R Pebody (richard.pebody@phe.gov.uk) ${ }^{1}$

1. Respiratory Diseases Department, Public Health England (PHE), Colindale, London, United Kingdom

2. Respiratory Virus Unit, Virus Reference Department, Public Health England(PHE) Microbiology Services, London, United Kingdom

3. Bristol Public Health Laboratory, Public Health England (PHE), Bristol, United Kingdom

Citation style for this article:

Zhao H, Green H, Lackenby A, Donati M, Ellis J, Thompson C, Bermingham A, Field J, SebastianPillai P, Zambon M, Watson JM, Pebody R. A new laboratory-based surveillance system (Respiratory DataMart System) for influenza and other respiratory viruses in England: results and experience from 2009 to 2012 . Euro Surveill. 2014;19(3):pii=20680. Available online: http://www.eurosurveillance.org/ViewArticle.aspx?Articleld=20680

During the 2009 influenza $A\left(\mathrm{H}_{1} \mathrm{~N}_{1}\right)$ pandemic, a new laboratory-based virological sentinel surveillance system, the Respiratory DataMart System (RDMS), was established in a network of 14 Health Protection Agency (now Public Health England (PHE)) and National Health Service (NHS) laboratories in England. Laboratory results (both positive and negative) were systematically collected from all routinely tested clinical respiratory samples for a range of respiratory viruses including influenza, respiratory syncytial virus (RSV), rhinovirus, parainfluenza virus, adenovirus and human metapneumovirus (hMPV). The RDMS also monitored the occurrence of antiviral resistance of influenza viruses. Data from the RDMS for the 2009-2012 period showed that the 2009 pandemic influenza virus caused three waves of activity with different intensities during the pandemic and post pandemic periods. Peaks in influenza $A\left(\mathrm{H}_{1} \mathrm{~N}_{1}\right)$ pdmog positivity (defined as number of positive samples per total number of samples tested) were seen in summer and autumn in 2009, with slightly higher peak positivity observed in the first post-pandemic season in 2010/2011. The influenza $A\left(\mathrm{H}_{1} \mathrm{~N}_{1}\right)$ pdmog virus strain almost completely disappeared in the second postpandemic season in 2011/2012. The RDMS findings are consistent with other existing community-based virological and clinical surveillance systems. With a large sample size, this new system provides a robust supplementary mechanism, through the collection of routinely available laboratory data at minimum extra cost, to monitor influenza as well as other respiratory virus activity. A near real-time, daily reporting mechanism in the RDMS was established during the London 2012 Olympic and Paralympic Games. Furthermore, this system can be quickly adapted and used to monitor future influenza pandemics and other major outbreaks of respiratory infectious disease, including novel pathogens.

\section{Introduction}

The long-standing national laboratory surveillance system in England known as LabBase [1] collects positive reports of detections of a wide range of infectious pathogens, but does not collect negative results. Two sentinel general practitioner virological surveillance schemes, the Royal College of General Practitioners (RCGP) and Public Health England (PHE, the former Health Protection Agency (HPA) is part of PHE from April 2013) scheme (RCGP/PHE scheme) [2-5], and the PHE and Regional Microbiological Network (RMN) swabbing scheme (PHE/RMN scheme) [6], have been in operation in England since the early 1990 s to monitor influenza activity in primary care settings (i.e. community settings) during the winter period. As part of strengthening respiratory virus surveillance in response to the 2009 influenza $A\left(\mathrm{H}_{1} \mathrm{~N}_{1}\right)$ pandemic [7-10], a new laboratory-based respiratory virus surveillance system, the Respiratory DataMart System (RDMS), was developed in England in 2009. This laboratory surveillance system was initially established to collect both positive and negative results for the specific detection and confirmation of infection with influenza $A\left(\mathrm{H}_{1} \mathrm{~N}_{1}\right)$ pdmog virus from a network of laboratories in England using a newly implemented PCR assay [11]. The system was later extended to facilitate the monitoring not only of influenza virus but also of other major respiratory viruses, including respiratory syncytial virus (RSV), human metapneumovirus (hMPV), rhinovirus, parainfluenza viruses, and adenovirus. The primary objective of the RDMS as a new surveillance system is to alert relevant stakeholders on the incidence trends of these viruses. The RDMS operates all year round collecting results of 
all routinely tested respiratory clinical samples from participating laboratories. These samples have been taken from both primary and secondary healthcare settings. This paper describes this new RDMS system and results from data collected during the pandemic and post-pandemic periods between 2009 and 2012. It also provides a preliminary evaluation of this new system in comparison with other existing surveillance systems.

\section{Methods}

A network of 14 laboratories representing all nine regions of England currently participates in the RDMS, including the national reference laboratory (the PHE Respiratory Virus Unit (PHE-RVU) of the Virus Reference Department, Colindale, London), all major PHE regional laboratories and four local National Health Service (NHS) laboratories. All participating laboratories, except the PHE-RVU, provide routine respiratory virus diagnostics service for their affiliated major regional or local hospitals.

Laboratory tests are requested by clinicians in charge of patient care. Clinicians decide the test each patient needs, and which types of samples need to be taken, and when. The most common sample types reported to this system are nasopharyngeal aspirate, tracheal secretion and nasal and throat swab. This system does not collect data on patients' clinical condition or case definitions used by clinicians.

All participating laboratories detect influenza, RSV, rhinovirus, parainfluenza 1-4 and hMPV using reverse transcription real-time polymerase chain reaction (rRT$P(R)$, and adenovirus using real-time PCR. All laboratories validated their assays appropriately. Quality assurance is achieved by participation in External Quality Assurance (EQA) programmes.

All respiratory virus test results are submitted to RDMS every week throughout the year. Both positive and negative results are submitted. The test results of influenza $\mathrm{A}\left(\mathrm{H}_{1} \mathrm{~N}_{1}\right)$ pdmog were collected and stored in another system before the establishment of the RDMS in November 2009. These results were transferred into the RDMS. Since November 2009, results for other respiratory viruses tested in routine respiratory virus $P C R$ systems (including RSV, rhinovirus, parainfluenza 1-4, adenovirus and hMPV) have also been included in the RDMS.

Influenza A subtyping results were reported by 10 of the 14 participating laboratories; one laboratory carried out the test but results have not yet been reported, and the remaining three laboratories did not perform influenza subtyping.

As part of daily respiratory virus surveillance for the London 2012 Olympic and Paralympic Games, a subset of seven major participating laboratories undertook daily data submission from April to September 2012 to feed into the internal PHE's daily national Olympic situation reports produced during the Games period between July and September 2012.

Although participating laboratories employ different laboratory information management systems (such as Winpath, Telepath and Apex) and data codes, a standard common set of data items has been defined, including items such as sex, date of birth, sample date and virological test results for each virus. Programmes have been developed which standardise these data for combination in a central database. Data submission is carried out through a secure online data submission tool. De-duplication is carried out during data importation using a six-week episode period, which is consistent with the PHE national laboratory database, LabBase [1], and is intended to capture all possible test results relating to the same episode of a respiratory infection.

Submitted data contain all test results carried out by the 14 participating laboratories for all respiratory samples taken from hospital inpatients, hospital outpatients, and patients in primary care settings. Samples collected through both the RCGP/PHE and PHE/RMN sentinel general practitioners (GP) schemes from patients in the community are also included in the RDMS.

The proportion of samples positive (positivity) for viruses under surveillance is calculated (based on weekly samples tested) by virus type and by age group $(55,5-4,15-44,45-64$ and $65+$ years) using weekly number of positive detections divided by the weekly total number of samples tested. Positivity was not calculated when the sample size was less than 10 in our study. A three-week moving average of the positivity is used to smooth the random fluctuation of the weekly positivity. The data are analysed to determine trends and predominant virus types. To compare the RDMS with other existing surveillance systems, the overall proportion of samples positive (positivity) for influenza during the 2010/11 and 2011/12 winter seasons are used with the results from other influenza surveillance systems including the weekly influenza-like illness (ILI) GP consultation rates (weekly number of ILI patients per 100,000 GP registered population) reported from the Research \& Surveillance Centre of the Royal College of General Practices (RCGP) [5, 12-15]; the proportion of total weekly calls made through to the NHS Direct (NHSD) telephone helpline in England for fever in 5-14 years (NHSD Fever) [16-19], and the community influenza positivity using the combined overall proportion of samples positive for influenza (including all types/ subtypes of influenza) from the two community-based GP sentinel virological surveillance schemes (the RCGP/PHE scheme [2-5] and the PHE/RMN scheme [6]). The original weekly data values for various indicators from other systems were available and examined in comparison with this new RDMS system for season start, peak time and trend. 
Weekly number of samples submitted to Respiratory DataMart System during influenza and non-influenza seasons, England, April 2009-July 2012

\begin{tabular}{|l|c|c|}
\hline Study period & $\begin{array}{c}\text { Range of sample numbers per week during } \\
\text { influenza season (week 40-week 20) }\end{array}$ & $\begin{array}{c}\text { Range of sample numbers per week during } \\
\text { non-influenza season (week 21-week 39) }\end{array}$ \\
\hline Apr 2009-Sep 2009 & NA & $600-10,000^{\text {a }}$ \\
\hline Oct 2009-Sep 2010 & $400-4,000$ & $300-500$ \\
\hline Oct 2010-Sep 2011 & $500-6,000$ & $200-500$ \\
\hline Oct 2011-Jul 2012 & $500-1,000$ & $200-500$ \\
\hline
\end{tabular}

NA: not applicable.

a First wave of the 2009 influenza pandemic.

A subset of four laboratories, including the PHE-RVU, also submit influenza antiviral susceptibility testing results for oseltamivir (from all these four laboratories) and zanamivir (from the PHE-RVU only). Three regional laboratories perform a real-time genotyping PCR for rapid discrimination of a single nucleotide change (tyrosine to histidine at position $275 ; \mathrm{H} 275 \mathrm{Y}$ mutation) in the neuraminidase gene of influenza $A\left(\mathrm{H}_{1} \mathrm{~N}_{1}\right)$ pdmog viruses that confers oseltamivir (Tamiflu) resistance (methodology available on request). PHE-RVU confirm oseltamivir-resistant virus detections from the regional laboratories and perform additional screening of $A\left(\mathrm{H}_{1} \mathrm{~N}_{1}\right)$ pdmog viruses for the $\mathrm{H}_{275} \mathrm{Y}$ mutation using pyrosequencing methodology as previously described [20]. In addition, PHE-RVU analyses virus isolates with sufficient neuraminidase activity phenotypically for susceptibility to oseltamivir and zanamivir, by a fluorescence-based neuraminidase enzyme inhibition assay, described previously [21].

A weekly report is produced, based on the RDMS data of influenza and other respiratory viruses, to track the weekly number of positive results and weekly proportion of positives (positivity) by sampling week, age group and virus type which is summarised in the PHE Weekly National Influenza Report [22] and in the accompanying graph collection on the PHE website [23].

The process of data collection, management and application for RDMS has been approved by the National Information Governance Board for Health and Social Care.

\section{Results}

The number of respiratory samples reported to RDMS from participating laboratories is summarised in Table 1.

The sample source data is currently only available from three participating laboratories, including the national reference laboratory (PHE-RVU) and two regional laboratories. This represents 43,949 of all 201,537 samples collected from all participating laboratories in the RDMS up to week 27 2012. The sample source data from two regional laboratories indicates that the biggest proportion of RDMS samples (68.3\%) were from patients admitted into secondary care settings (mainly hospital inpatients), with $3.0 \%$ from primary care settings and the rest (28.7\%) from other sources (unspecified or unnamed sources). However, the sample source data from PHE-RVU shows that the majority of samples $(88.7 \%)$ were from primary care settings, with only $11.3 \%$ from secondary care settings. Information is not currently available on sample source from the remaining 11 laboratories.

The RDMS database was set up as a weekly reporting system, although seven laboratories reported daily data during the 2012 Olympic and Paralympic Games period. The reporting delay time from sampling date to the date of the results reported to the RDMS was examined and showed that $63.6 \%$ of sample results were reported to the system within a week of sampling, $95.8 \%$ within two weeks and $98.1 \%$ within three weeks.

Results for influenza by type and subtype are shown in Figures $1 \mathrm{a}$ (overall) and $1 \mathrm{~b}$ (by age group) from the start of the 2009 pandemic in April 2009 to July 2012 (week 27 2012), including the two pandemic waves of the 2009 influenza pandemic. Figures $2 a$ and $2 b$ show the number of positive detections and proportion of samples positive for other respiratory viruses (data available from November 2009) by sampling week and by age group, respectively, to week 272012.

The first wave of the 2009 pandemic occurred between late April 2009 and August 2009, with the peak weekly influenza positivity at $35.1 \%$ in June 2009. The second pandemic wave occurred between August 2009 and February 2010 with the peak positivity at $34.2 \%$ in October 2009 (Figure 1a). During the 2010/11 season, there was a single peak of activity with overall influenza positivity reaching $38.4 \%$, and a low level of influenza B co-circulating (Figure 1a). During the 2011/12 season, the predominant strain in circulation was influenza $\mathrm{A}\left(\mathrm{H}_{3}\right)$ at a low level, reaching a peak of $17.6 \%$ in week 9 2012, with very few sporadic influenza $A\left(\mathrm{H}_{1} \mathrm{~N}_{1}\right)$ pdmog viruses and some influenza $B$ viruses (Figure 1a). Figure $1 \mathrm{~b}$ shows a different age distribution between 2009/10, 2010/11 and 2011/12 seasons. During the 2009/10 pandemic period, the age group with the highest positivity was children aged between 
Number of positive tests and proportion of positive results for influenza by influenza subtype and week (A) and proportion of samples positive for influenza by age group and week (B) England, April 2009-July 2012*

A
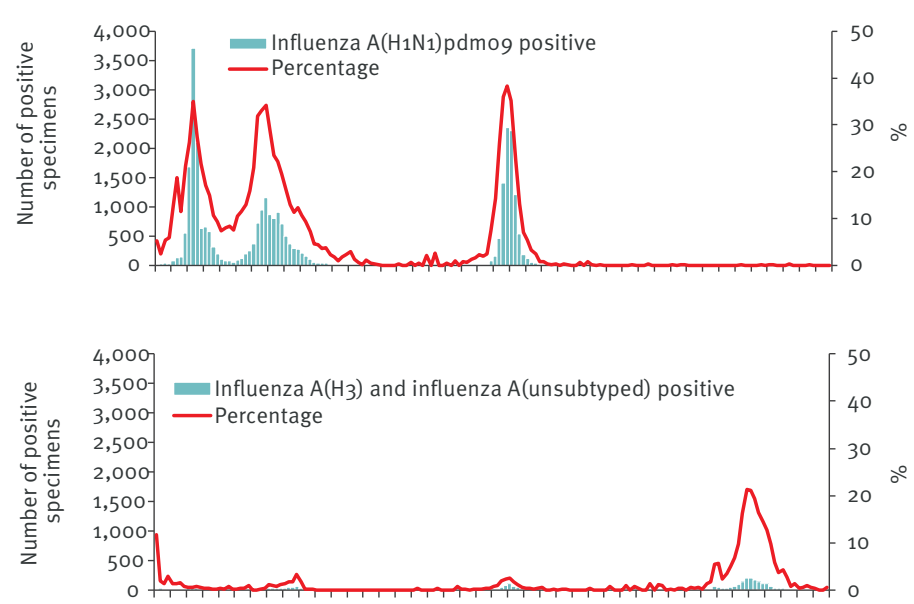

B
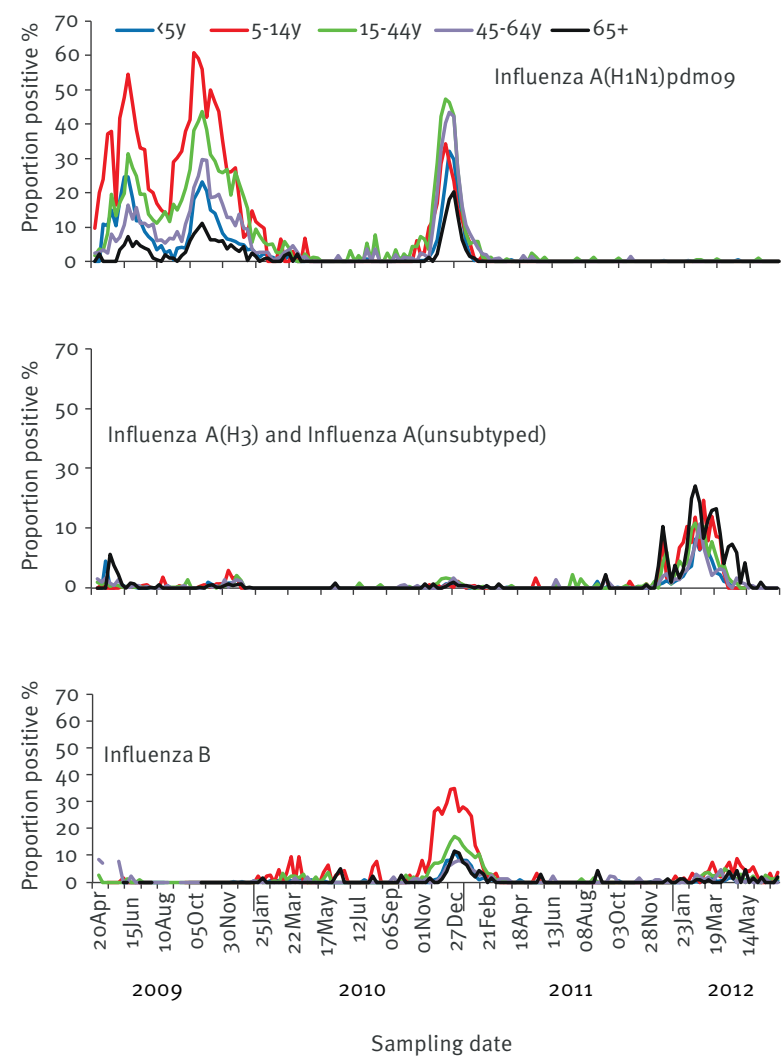

during December 2009 and December 2010. Clear seasonality was also observed for hMPV with higher positivity between February and April, and lower positivity during summer and autumn.

The three-week moving averages of the weekly proportions of samples positive for other respiratory viruses by age group are shown in Figure 2 b. Patients under 5 years of age usually had the highest proportion positive for all of these respiratory viruses. Patients aged 5-14 years also had a higher proportion positive for adenovirus and rhinovirus.

Results from the daily data submission had been extracted and used to produce the daily PHE national situation report (internal report) during the period of the 2012 Olympic and Paralympic Games. The numbers of positive detections of influenza by type and subtype and any significant findings from the daily data for other respiratory viruses were reported each day during the Games period.

In addition to the proportion positive results described above, the weekly proportion negative among these samples was also examined and shown in Figure 3. 


\section{FIGURE 2}

Number of positive tests per week and three-week moving average of proportion of positive results for major respiratory viruses other than influenza (A) and three-week moving average of the weekly proportion of samples positive for major respiratory viruses other than influenza by age group (B) England, November 2009 - July 2012*

A
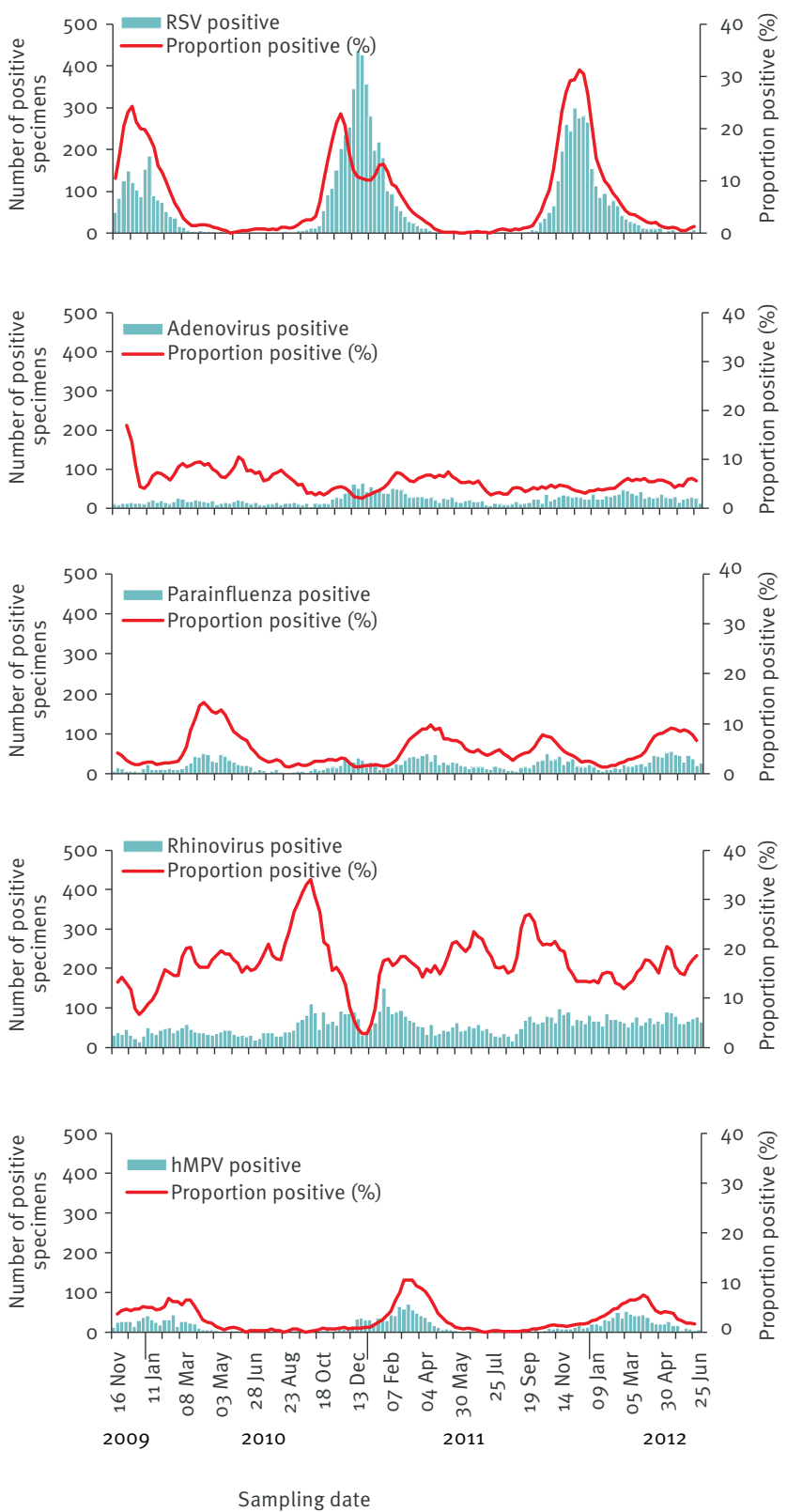

hMPV: human metapneumovirus; RSV: respiratory syncytial virus.
B
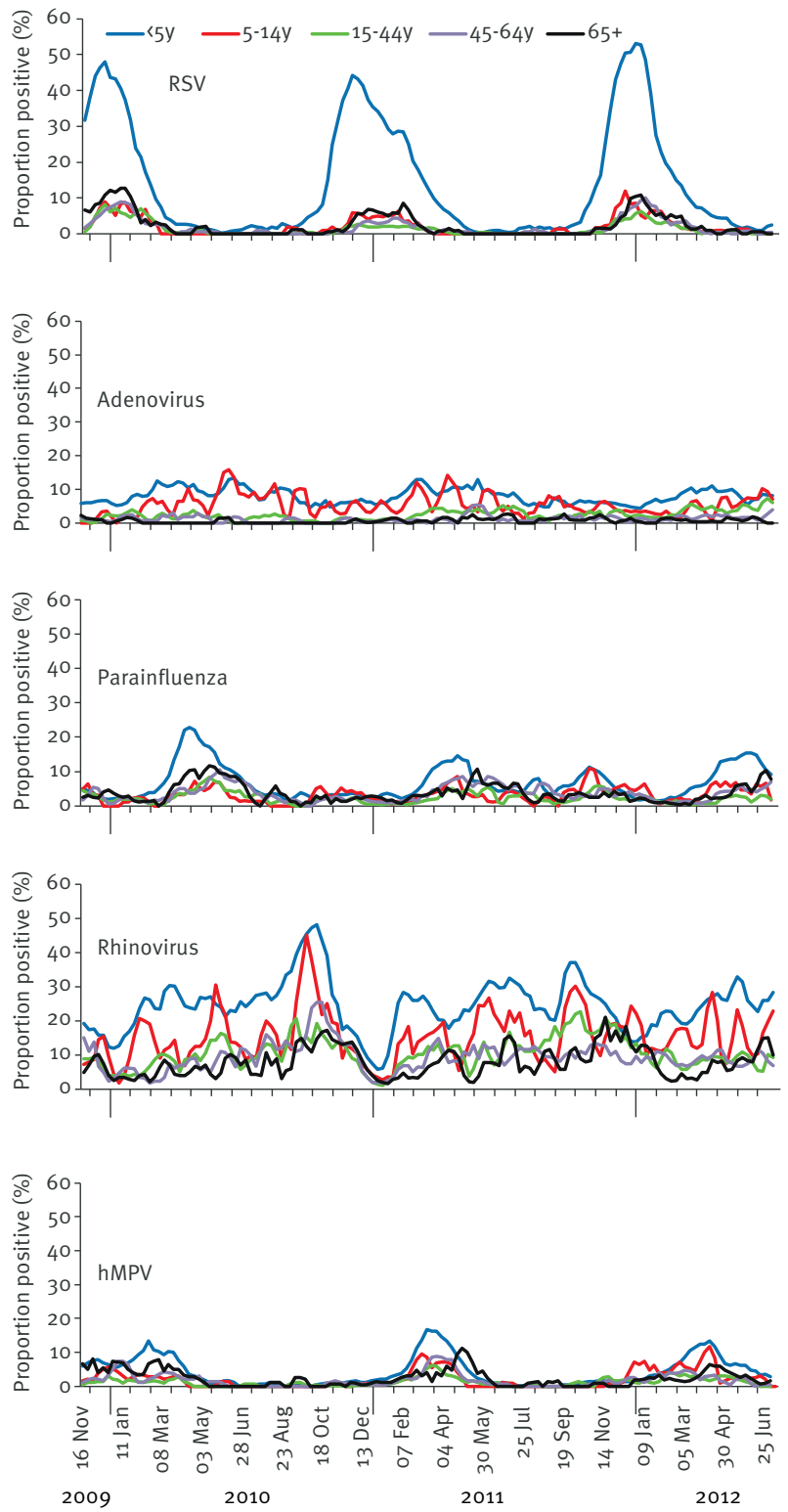

Sampling date 
Proportion of samples testing negative for influenza A and B, respiratory syncytial virus, adenovirus, rhinovirus, parainfluenza viruses and human metapneumovirus: results of Respiratory DataMart System, England, November 2009July 2012

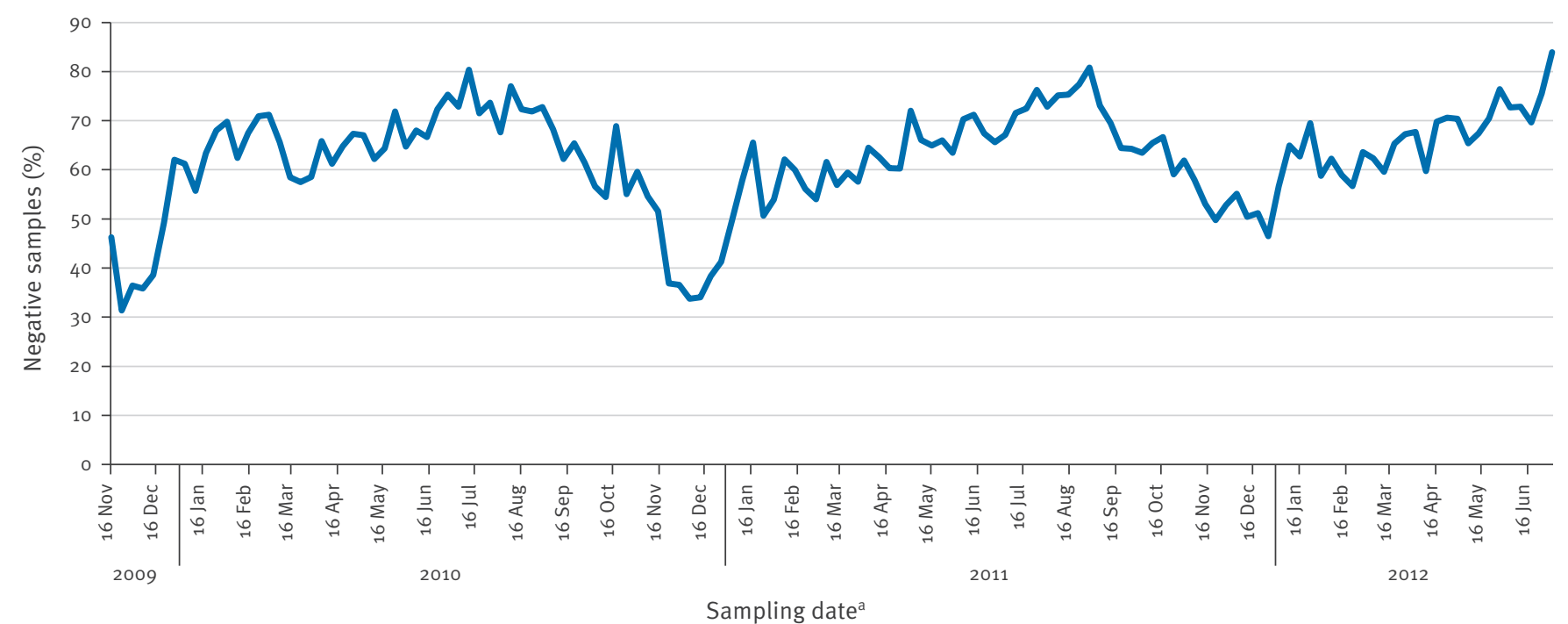

a Samples were tested weekly.

This figure shows that during the study period, the proportion of samples testing negative for any intended respiratory viruses included in the RDMS system, i.e. influenza $A$ and $B, R S V$, adenovirus, rhinovirus, parainfluenza and hMPV, demonstrated a clear seasonality with low proportion negative observed during the winter period and high proportion negative during the summer and autumn period.

A comparison of the results of RDMS with other existing influenza activity surveillance systems in England is shown in Figure 4. The RDMS weekly influenza proportion positivity was lower but demonstrated a steady trend compared with the community influenza positivity (Figure 4). The first signals of the start of the 2010/11 season's influenza activity were seen from the NHSD fever calls in the 5-14 year-olds which started to increase from week 452010 and rose above the season's threshold level of $9 \%$ from week 472010 , together with the community influenza positivity and RDMS influenza positivity which started to increase significantly from week 472010 and peaked between weeks 50-52 2010. The RCGP consultation rates increased significantly from week 492010 and peaked in week 51 2010, with a second peak in week 12011 (which may be due to delayed consultation over the Christmas and New Year holiday). The 2011/12 season's influenza activity was low and came late, increasing slowly from week 3 2012, with the first increase observed from the RDMS influenza positivity in week 22012 followed by the community influenza positivity one week later. Positivity peaked during weeks 72012 and 82012.
Table 2 shows the antiviral test results reported to RDMS by four participating laboratories performing antiviral susceptibility testing, including PHE-RVU, during the 2010/11 and 2011/12 seasons. Three regional laboratories reported oseltamivir susceptibility testing data, accounting for $25.7 \%$ of all tests (screening for the $\mathrm{H}_{275} \mathrm{Y}$ mutation in the neuraminidase gene of influenza $\mathrm{A}\left(\mathrm{H}_{1} \mathrm{~N}_{1}\right)$ pdmog viruses), although the majority of antiviral susceptibility test results for influenza $A$ and all test results for influenza B came from the PHERVU which carries out UK-wide confirmatory testing for any oseltamivir resistance detections and all zanamivir resistance tests. A total of 59 (3.3\%) oseltamivir-resistant influenza $A\left(\mathrm{H}_{1} \mathrm{~N}_{1}\right)$ pdmog viruses were detected during the 2010/11 season in the UK. No oseltamivir -or zanamivir-resistant influenza virus was detected up to week 272012 for the 2011/12 season. In addition, 63 of the influenza $\mathrm{A}\left(\mathrm{H}_{3}\right)$ viruses detected during the 2011/12 season have also been tested against $M_{2}$ inhibitors (amantadine and rimantadine) by the PHE-RVU. All are resistant, with the $S_{31} \mathrm{~N}$ substitution, which is as expected for the circulating strain during that period.

\section{Discussion}

This article presents the findings of a new laboratory-based respiratory virus surveillance system, the Respiratory DataMart System (RDMS), which was developed and implemented during the influenza $A\left(\mathrm{H}_{1} \mathrm{~N}_{1}\right)$ 2009 pandemic in England. The system provides useful information in a timely fashion which has contributed to describing the epidemiology of influenza and other respiratory viruses during the 2009 pandemic and the two post-pandemic influenza seasons (2010/11 and $2011 / 12)$. The system is also able to monitor a range 
Comparisons between Respiratory DataMart System and other monitoring systems for respiratory viruses by week, England, 2010/11 and 2011/12

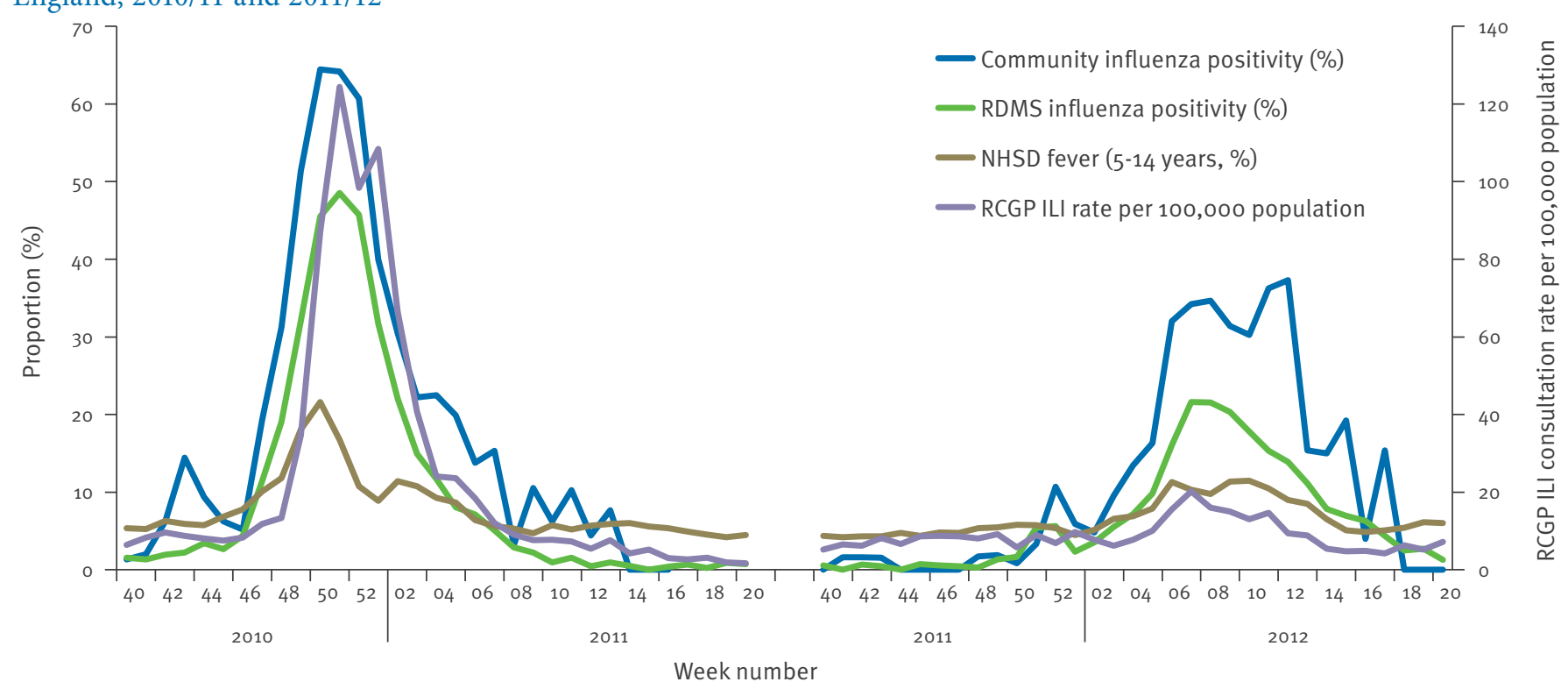

ILI: influenza-like illness; NHSD: NHS Direct; RCGP: Centre of the Royal College of General Practices; RMDS: Respiratory DataMart System.

of other respiratory viruses and influenza antiviral susceptibility. Comparison of this new system (RDMS) with other established surveillance systems shows that it consistently enables us to detect the start of the influenza season at an early time. The system was also successfully used for near real-time, daily surveillance during the London 2012 Olympic and Paralympic Games.

Data from the RDMS system show that the 2009 pandemic virus in 2009/10 had two waves, followed by a further post-pandemic wave in 2010/11, with the most affected age group shifting from 5-14 years in 2009/10 to $15-44$ years in $2010 / 11$. In the $2011 / 12$ season which followed, the 2009 pandemic virus circulated very little, with low levels of influenza $\mathrm{A}\left(\mathrm{H}_{3}\right)$ as the predominant strain. This phenomenon has been observed elsewhere in Europe [24] and very low levels of the influenza $A\left(\mathrm{H}_{1} \mathrm{~N}_{1}\right)$ pdmog strain were reported in the northern hemisphere except for Mexico, where it was the predominant strain. Overall levels were low in the United States and Canada during the 2011/12 season [25]. The RDMS system was also able to provide the data to the level of influenza subtype, which is critical in order to understand the epidemiology of influenza each season.

It may also be possible to use RDMS to describe the epidemiology of a range of other respiratory viruses. RSV was the most notable of these, with a high number of positive samples detected and high positivity. The marked regular seasonality of RSV activity was clearly displayed over the study period, peaking each November/December, with the most affected population being children aged under five years. These features of seasonality and the different impact on various age groups have previously been recognised [2628]. Clear seasonality was also found for parainfluenza viruses peaking in April to May and hMPV in February to April each year. Children under the age of five years were predominantly affected by both viruses. These findings were also consistent with previous studies [27-31]. No clear seasonality was found for adenovirus and rhinovirus, with rhinovirus being the second most reported virus in the RDMS following RSV and mainly affecting children under five years old.

Surveillance for influenza is common practice in most European countries [32] but routine surveillance for other respiratory viruses is not common. The RDMS thus provides a new mechanism to monitor the epidemiology of acute respiratory viral infections in a timely fashion.

The number of laboratories that submit data varies by week, which will have an impact on the number of samples received, an issue also seen with LabBase. Therefore, the absolute number of positive detections each week may not be a reliable indicator for disease surveillance purposes. The proportion of samples positive, however, can be a useful additional indicator in situations where the sample size is large enough. There was a drop in the proportion of tests positive in early June 2009 for influenza $A\left(\mathrm{H}_{1} \mathrm{~N}_{1}\right)$ pdmo9 which occurred (Figures 1a and 1b) a few weeks before the first peak in number of cases in late June 2009. This was due to a significantly increased number of samples being tested in that week, especially in the 5-14 
TABLE 2

Influenza antiviral susceptibility testing results for oseltamivir and zanamivir, United Kingdom, 2010/11 and 2011/12

\begin{tabular}{|c|c|c|c|c|c|c|c|c|c|c|c|c|c|}
\hline \multirow{3}{*}{ Laboratory } & \multirow{3}{*}{$\begin{array}{l}\text { Influenza } \\
\text { type / } \\
\text { subtype }\end{array}$} & \multicolumn{6}{|c|}{ Oseltamivir } & \multicolumn{6}{|c|}{ Zanamivir } \\
\hline & & \multicolumn{2}{|c|}{ Resistant } & \multicolumn{2}{|c|}{ Sensitive } & \multicolumn{2}{|c|}{$\%$ Resistant } & \multicolumn{2}{|c|}{ Resistant } & \multicolumn{2}{|c|}{ Sensitive } & \multicolumn{2}{|c|}{$\%$ Resistant } \\
\hline & & $2010 / 11$ & $2011 / 12$ & $2010 / 11$ & $2011 / 12$ & $2010 / 11$ & $2011 / 12$ & $2010 / 11$ & $2011 / 12$ & $2010 / 11$ & $2011 / 12$ & $2010 / 11$ & $2011 / 12$ \\
\hline \multirow{3}{*}{$\begin{array}{l}\text { HPA-RVU } \\
\text { (UK-wide) }\end{array}$} & $\mathrm{A}\left(\mathrm{H}_{3} \mathrm{~N}_{2}\right)$ & 0 & 0 & 4 & 190 & \begin{tabular}{|l|}
0 \\
\end{tabular} & 0 & 0 & \begin{tabular}{|l|}
0 \\
\end{tabular} & \begin{tabular}{|l|}
4 \\
\end{tabular} & 190 & 0 & 0 \\
\hline & $\begin{array}{l}\mathrm{A}(\mathrm{H} 1 \mathrm{~N} 1) \\
\text { pdmo9 }\end{array}$ & 59 & 0 & 1,382 & 5 & 4.1 & 0 & 0 & 0 & 155 & 1 & 0 & 0 \\
\hline & B & 0 & 0 & 317 & 10 & 0 & 0 & 0 & 0 & 317 & 10 & 0 & 0 \\
\hline Manchester & $\begin{array}{l}\mathrm{A}\left(\mathrm{H}_{1} \mathrm{~N} 1\right) \\
\text { pdmo9 }\end{array}$ & $1^{\mathrm{a}}$ & 0 & 380 & 1 & 0.3 & 0 & NT & NT & NT & NT & NT & NT \\
\hline Birmingham & $\begin{array}{l}\mathrm{A}\left(\mathrm{H}_{1} \mathrm{~N}_{1}\right) \\
\text { pdmo9 }\end{array}$ & $1^{\mathrm{a}}$ & 0 & 40 & 0 & 2.4 & NA & NT & NT & NT & NT & NT & NT \\
\hline Southampton & \begin{tabular}{|l|}
$\mathrm{A}(\mathrm{H} 1 \mathrm{~N} 1)$ \\
pdmo9
\end{tabular} & $1^{\mathrm{a}}$ & 0 & 46 & 2 & 2.1 & 0 & NT & NT & NT & NT & NT & NT \\
\hline \multirow{3}{*}{$\begin{array}{l}\text { Total }^{\mathrm{b}} \\
\text { (UK-wide) }\end{array}$} & $\mathrm{A}\left(\mathrm{H}_{3} \mathrm{~N}_{2}\right)$ & 0 & 0 & 4 & 190 & 0 & 0 & 0 & 0 & 4 & 190 & 0 & 0 \\
\hline & $\begin{array}{l}\mathrm{A}\left(\mathrm{H}_{1} \mathrm{~N}_{1}\right) \\
\mathrm{pdmo9}\end{array}$ & 59 & 0 & 1,812 & 8 & 3.2 & 0 & 0 & 0 & 155 & 1 & o & 0 \\
\hline & B & 0 & 0 & 317 & 10 & 0 & 0 & 0 & 0 & 317 & 10 & 0 & 0 \\
\hline
\end{tabular}

HPA - RVU: Health Protection Agency - Respiratory Virus Unit; NA: not applicable; NT: not tested; UK: United Kingdom.

a This sample is also included in the HPA-RVU total.

${ }^{\mathrm{b}}$ The figures in the total rows are figures after de-duplication.

year age group, while the number of positive samples only increased slightly over the same time period. This increased testing probably reflected the intensive pandemic case finding practice applied for early detection of suspected cases during the containment phase in the early stage of the 2009 pandemic in England [33] and highlights the value of the proportion positive indicator.

RDMS relies on patients being sufficiently unwell to seek medical care and being considered clinically suitable for testing. A number of other viral and bacterial respiratory pathogens are currently either not included in the test screen or not reported to the RDMS (e.g. Mycoplasma pneumoniae). Inclusion of these would affect the overall positivity of pathogen detection. Thus if numbers tested increased significantly but the proportion positive fell, this might reflect poor case finding but could also reflect an alternative respiratory pathogen being responsible for the presumed infection. The seasonality shown in the analyses of the proportion negative may indicate some contribution from other pathogens not included in the RDMS database, although this seasonality may be due mainly to the seasonal variations of respiratory viruses already included in the RDMS.

Daily surveillance data is in high demand, especially during events with high public health importance such as the London 2012 Olympic and Paralympic Games, and influenza pandemic or epidemic periods. It is a great advantage for RDMS to be able to provide daily laboratory data to monitor influenza and other respiratory viruses. This daily surveillance operation can pick up early signs of increased activity of these viruses covered by the system and thus the ability to undertake prompt response and appropriate actions.

Comparing this new system (RDMS) with other established surveillance systems during the 2010/11 and 2011/12 influenza seasons shows that although the peak positivity values from RDMS are lower than from the community-based GP sentinel virological system (probably due in part to a much larger sample size in the RDMS), RDMS generally provides an early indication of the start of the influenza season. Figure $3 \mathrm{dem}$ onstrates that the RDMS data could act as an additional important source using routinely available laboratory data to detect the beginning of increased activity of influenza (and other respiratory viruses), and to track their epidemic trends.

The main strengths of the RDMS are that it collects both positive and negative test results all year round for influenza and several other common respiratory viruses. It is geographically representative: participating laboratories include all major regional laboratories plus several local laboratories across the country, with large sample numbers from both community and hospital settings. Similar positive numbers of influenza virus are reported to the RDMS and to the national LabBase database, which collects results from about 
100 laboratories across the country, which indicates the completeness of reporting to the RDMS.

There are some limitations for this study. The lack of information on the source of the sample (i.e. primary vs secondary care settings) from many of the participating laboratories is one of them. However, the two regional laboratories where sample source data were available are very similar in terms of sample referral and test procedures, compared with the remaining 11 regional and local NHS laboratories. We therefore believe that the sample source results from these two regional laboratories should be generalisable, i.e. the majority of the samples for the regional and local NHS laboratories are from secondary care settings. Efforts are continuing to capture sample source information from the remaining laboratories.

This study covers the RDMS operation period for three influenza seasons between 2009 and 2012, during which time only the $2011 / 12$ season was dominated by influenza $\mathrm{A}\left(\mathrm{H}_{3}\right)$. Therefore, longer period data from this system will be needed to further evaluate this system's ability to monitor the usual influenza $\mathrm{A}\left(\mathrm{H}_{3}\right)$ circulating situations, compared with other surveillance systems.

Virological surveillance of influenza and other respiratory viruses is crucial in order to determine which viruses are actually circulating in a population, and their timing, trend and impact. Using routinely available hospital laboratory data at minimum extra cost is an important addition to the current respiratory virus surveillance system. This approach has been tried in the US, which showed a good correlation with other influenza surveillance data during the 2009/10 pandemic period [34]. Hospital laboratory test results have been fed into some weekly influenza surveillance reports in some other European countries such as France and Denmark [35]. The RDMS has representative coverage of England, captures high volumes of samples and provides timely reports and feedback to data providers and stakeholders. It provides an important supplement to the routine influenza surveillance systems for both pandemic and seasonal influenza. With the accumulation of further years of data, thresholds and exceedance reports for each virus will become established. Furthermore, the RDMS can be easily adapted to add emerging pathogens, such as the novel coronavirus (MERS-CoV) or a new influenza pandemic virus.

\section{Acknowledgments}

We would like to thank Neill Keppie and Nick Richardson for setting up and providing technical support for this system and all contributing PHE and NHS laboratories

\section{*Erratum:}

Figures 1 and 2 were erroneously swapped. This was corrected on 19 May 2014.
References

1. Grant AD, Eke E. Application of information technology to the laboratory reporting of communicable disease in England and Wales. Commun Dis Rep CDR Rev. 1993;3(6):R75-8.

2. Fleming DM, Chakraverty P, Sadler C, Litton P. Combined clinical and virological surveillance of influenza in winters of 1992 and 1993-4. BMJ. 1995;311(7000):290-1. http://dx.doi. org/10.1136/bmj.311.7000.290

3. Boon AC, French AM, Fleming DM, Zambon MC. Detection of influenza A subtypes in community-based surveillance. J Med Virol. 2001;65(1):163-70. http://dx.doi.org/10.1002/jmv.2016

4. Ellis JS, Fleming DM, Zambon MC. Multiplex reverse transcription-PCR for surveillance of influenza A and B viruses in England and Wales in 1995 and 1996. J Clin Microbiol. 1997;35(8):2076-82.

5. Fleming DM, Elliot AJ. Lessons from 40 years' surveillance of influenza in England and Wales. Epidemiol Infect. 2008;136(7):866-75. http://dx.doi.org/10.1017/ So950268807009910

6. Joseph CA. Virological surveillance of influenza in England and Wales: results of a two year pilot study 1993/94 and 1994/95. PHLS Influenza Subcommittee. Commun Dis Rep CDR Rev. 1995; :R141-5.

7. Health Protection Agency and Health Protection Scotland New Influenza $A\left(\mathrm{H}_{1} \mathrm{~N}_{1}\right)$ Investigation Teams. Epidemiology of new influenza $A\left(\mathrm{H}_{1} \mathrm{~N}_{1}\right)$ in the United Kingdom, April-May 2009. Euro Surveill. 2009;14(19). pii:19213.

8. Health Protection Agency, Health Protection Scotland, National Public Health Service for Wales, and HPA Northern Ireland Swine influenza investigation teams. Epidemiology of new influenza $A\left(\mathrm{H}_{1} \mathrm{~N}_{1}\right)$ virus infection, United Kingdom, April-June 2009. Euro Surveill. 2009;14(22):pii:19232.

9. Pebody RG, McLean E, Zhao H, Cleary P, Bracebridge S, Foster $\mathrm{K}$, et al. Pandemic Influenza $\mathrm{A}\left(\mathrm{H}_{1} \mathrm{~N}_{1}\right) 2009$ and mortality in the United Kingdom: risk factors for death, April 2009 to March 2010. Euro Surveill. 2010;15(20):pii: 19571

10. Health Protection Agency (HPA). Epidemiological report of pandemic $\left(\mathrm{H}_{1} \mathrm{~N}_{1}\right) 2009$ in the UK. London: HPA; 2010. Available from: http://www.hpa.org.uk/web/HPAwebFile/ HPAweb_C/1284475321350

11. Ellis J, Iturriza M, Allen R, Bermingham A, Brown K, Gray J, et al. Evaluation of four real-time PCR assays for detection of influenza $A\left(\mathrm{H}_{1} \mathrm{~N}_{1}\right) v$ viruses. Euro Surveill. 2009 Jun 4;14(22). pii: 19230.

12. Fleming DM, Crombie DL. The incidence of common infectious diseases: the weekly returns service of the Royal College of General Practitioners. Health Trends. 1985;17(1):13-6.

13. Fleming DM. Weekly Returns Service of the Royal College of General Practitioners. Commun Dis Public Health. 1999;2(2):96-100.

14. Fleming DM, Miles J. The representativeness of sentinel practice networks. J Public Health (Oxf). 2010;32(1):90-6. http://dx.doi.org/10.1093/pubmed/fdpo87

15. Elliot AJ, Fleming DM. Surveillance of influenza-like illness in England and Wales during 1966-2006. Euro Surveill 2006;11(10):249-50

16. Baker M, Smith GE, Cooper D, Verlander NQ, Chinemana F, Cotterill S, et al. Early warning and NHS Direct: a role in community surveillance? J Public Health Med. 2003;25(4):3628. http://dx.doi.org/10.1093/pubmed/fdgo96

17. Cooper DL, Verlander NQ, Elliot AJ, Joseph CA, Smith GE. Can syndromic thresholds provide early warning of national influenza outbreaks? J Public Health (Oxf). 2009;31(1):17-25. http://dx.doi.org/10.1093/pubmed/fdmo68

18. Cooper DL, Smith G, Baker M, Chinemana F, Verlander N, Gerard E, et al. National symptom surveillance using calls to a telephone health advice service--United Kingdom, December 2001-February 2003. MMWR Morb Mortal Wkly Rep. 2004;53 (Suppl):179-83.

19. Smith GE, Cooper DL, Loveridge P, Chinemana F, Gerard E, Verlander N. A national syndromic surveillance system for England and Wales using calls to a telephone helpline. Euro Surveill. 2006;11(12):220-4.

20. Esposito S, Molteni CG, Daleno C, Valzano A, Fossali E, Da Dalt $\mathrm{L}$, et al. Clinical importance and impact on the households of oseltamivir-resistant seasonal $\mathrm{A} / \mathrm{H}_{1} \mathrm{~N}_{1}$ influenza virus in healthy children in Italy. Virol J. 2010 Aug 26;7:202. doi: 10.1186/1743-422X-7-202. http://dx.doi. org/10.1186/1743-422X-7-202

21. Lackenby A, Democratis J, Siqueira MM, Zambon MC. Rapid quantitation of neuraminidase inhibitor drug resistance in influenza virus quasispecies. Antivir Ther. 2008;13(6):809-20.

22. Health Protection Agency (HPA). HPA Weekly National Influenza Report. Week 17, 2012. London: HPA; 2012. 
Available from: http://www.hpa.org.uk/webc/HPAwebFile/ HPAweb_C/1317133845350

23. Health Protection Agency (HPA). HPA Weekly National Influenza Graphs. Week 17, 2012. London: HPA; 2012. Available from: http://www.hpa.org.uk/webc/HPAwebFile/ HPAweb_C/1284475036543

24. European Centre for Disease Prevention and Control (ECDC). Weekly influenza surveillance overview, May 25, 2012 - Week 20. Stockholm:ECDC; 2012. Available from: http://www. ecdc.europa.eu/en/publications/Publications/Forms/ECDC_ DispForm.aspx?ID=892

25. Review of the 2011-2012 winter influenza season, northern hemisphere. Wkly Epidemiol Rec. 2012 Jun 15;87(24):233-40.

26. Goddard NL, Cooke MC, Gupta RK, Nguyen-Van-Tam JS. Timing of monoclonal antibody for seasonal RSV prophylaxis in the United Kingdom. Epidemiol Infect. 2007;135(1):159-62. http:// dx.doi.org/10.1017/S0950268806006601

27. Manoha C, Espinosa S, Ahob S-L, Huet F, Pothier P. Epidemiological and clinical features of hMPV, RSV and RVs infections in young children. J Clin Virol. 2007;38:221-6 http:// dx.doi.org/10.1016/j.jcv.2006.12.005

28. Mahalingam S, Schwarze J, Zaid A, Nissen M, Sloots T, Tauro S, et al. Perspective on the host response to human metapneumovirus infection: what can we learn from respiratory syncytial virus infections? Microbes Infect. 2006;8:285-93 http://dx.doi.org/10.1016/j.micinf.2005.07.001

29. Laurichesse H, Dedman D, Watson JM, Zambon MC. Epidemiological features of parainfluenza virus infections: laboratory surveillance in England and Wales, 19751997. Eur J Epidemiol. 1999;15(5):475-84. http://dx.doi. org/10.1023/A:1007511018330

30. Fry AM, Curns AT, Harbour K, Hutwagner L, Holman RC Anderson LJ. Seasonal trends of human parainfluenza viral infections: United States, 1990-2004. Clin Infect Dis. 2006 Oct 15;43(8):1016-22. http://dx.doi.org/10.1086/507638

31. Aberle JH, Aberle SW, Redlberger-Fritz M, Sandhofer MJ, Popow-Kraupp T. Human metapneumovirus subgroup changes and seasonality during epidemics. Pediatr Infect Dis J. 2010;29(11):1016-8.

32. European Centre for Disease Prevention and Control (ECDC. Weekly Influenza Surveillance Overview. 30 March, 2012. Stockholm: ECDC; 2012. Available from: http://ecdc.europa.eu/ en/publications/Publications/120330_SUR_Weekly_Influenza_ Surveillance_Overview.pdf

33. Health Protection Agency (HPA). The role of the Health Protection Agency in the 'containment' phase during the first wave of pandemic influenza in England in 2009. London: HPA; 2010. Available from: http://www.hpa.org.uk/webc/ HPAwebFile/HPAweb C/1274088320581

34. Chopra T, Binienda J, Mohammed M, Shyamraj R, Long P, Bach D, et al. A practical method for surveillance of novel $\mathrm{H}_{1} \mathrm{~N}_{1}$ influenza using automated hospital data. Infect Control Hosp Epidemiol. 2011;32(7):700-2. http://dx.doi.org/10.1086/660200

35. Statens Serum Institut (SSI). Luftvejsvirus undtagen influenza A og B. INFLUENZA-NYT. 2013;4. Danish. Available from: http:// www.ssi.dk/Aktuelt/Nyhedsbreve/INFLUENZA-NYT/2012-2013/ Uge\% 204\%20-\%202013/Uge\%204\%20-\%202013\%20-\%20 luftvejsvirus.aspx 